\title{
EFEITO DA SOLUBILIZAÇÃO E ENVELHECIMENTO NA MICROESTRUTURA E RESISTÊNCIA A CORROSÃO DA LIGA DE MAGNÉSIO AZ3 I
}

\author{
Camila Queiroz Correia Fernandes' \\ José Adilson de Castro \\ Elivelton Alves Ferreira ${ }^{2}$ \\ Darlene Souza da Silva Almeida² \\ Leonardo Martins da Silva
}

\section{Resumo}

Este trabalho apresenta um estudo comparativo da resistência a corrosão da liga de magnésio AZ3I tratada termicamente por solubilização e diferentes tempos de envelhecimento, submetida ao teste de polarização cíclica em solução de $3,5 \%$ de $\mathrm{NaCl}$. Amostras da liga foram solubilizadas a $440^{\circ} \mathrm{C}$ por 24 horas. Após a solubilização três amostras foram envelhecidas a $220^{\circ} \mathrm{C}$; por 2,6 e 12 horas, respectivamente. Foram realizadas análises microestruturais com a finalidade de compreender as alterações microestruturais advindas dos tratamentos térmicos realizados. As amostras foram submetidas ao ensaio de polarização cíclica com a finalidade de correlacionar o efeito das mudanças alcançadas pelos tratamentos térmicos na resistência à corrosão da liga de magnésio $A Z 3$ I. O comportamento das curvas de polarização cíclica foi semelhante para todas as amostras, sugerindo tendência a corrosão por pite. Não houve variação significativa nos valores de potencial de corrosão, sugerindo que os tratamentos térmicos aplicados não influenciaram a resistência a corrosão da liga.

Palavra-chave: Liga de magnésio; AZ3I; Corrosão; Polarização cíclica.

\section{COMPARATIVE BEHAVIOR OF MAGNESIUM ALLOY AZ3 I BY CYCLIC POLARIZATION TEST}

\begin{abstract}
This work presents a comparative study between the corrosion resistance of the magnesium alloy AZ3 I treated by solubilization and aging at different times, submitted to the cyclic polarization test in $3.5 \% \mathrm{NaCl}$ solution. Four samples were solubilized at $440^{\circ} \mathrm{C}$ for 24 hours. After solubilization, three samples were aged at $220^{\circ} \mathrm{C}$; one for 2 hours, another for 6 hours and a third for 12 hours. Microstructural analysis were carried out to identify microstructural changes obtained. The samples were submitted to the cyclic polarization test in order to correlate the effect of the changes achieved by heat treatments on the corrosion resistance of the magnesium alloy AZ3I. The cyclic polarization presents curves with similar behavior for the samples, indicating propensity to pitting corrosion. There were no significant variation in the values of corrosion potential, suggesting that the heat treatments applied did not influenced the corrosion resistance of the alloy. Keywords: Magnesium Alloy; AZ3 I; Corrosion resistance; Cyclic polarization.
\end{abstract}

\section{INTRODUÇÃO}

O magnésio e suas ligas têm sido apontados como alternativa para satisfazer exigências de indústrias que buscam constantemente por materiais que possuam ótima resistência mecânica e baixo peso, apresentando assim grande potencial em substituição aos aços e ligas de alumínio amplamente utilizadas em indústrias de transporte como automobilísticas e aeronáuticas. As ligas de magnésio possuem menor densidade (aproximadamente $1,74 \mathrm{~g} / \mathrm{cm}^{3}$ ) quando comparadas às ligas mais comuns utilizadas para a fabricação de elementos estruturais [I]. Contudo, apesar

'Engenharia Metalúrgica, Universidade Federal Fluminense - UFF, Volta Redonda, RJ, Brasil. E-mail: caqueirozc@gmail.com

${ }^{2}$ Universidade Federal Fluminense - UFF, Volta Redonda, RJ, Brasil.

2176-1523 (C) 2019 Associação Brasileira de Metalurgia, Materiais e Mineração. Publicado pela ABM. Este é um artigo de acesso aberto distribuído sob os termos da licença Creative Commons CC BY-NC-ND (Attribution-NonCommercial-NoDerivs) - https:// creativecommons.org/licenses/by-nc-nd/4.0\%. 
de seu grande potencial, as ligas deste grupo ainda não são amplamente utilizadas principalmente devido a sua baixa resistência a corrosão.

As primeiras ligas comercializadas de magnésio foram as com os elementos $\mathrm{Al}$ e $\mathrm{Zn}$ como elementos de liga e o sistema Mg-Al-Zn se mantém como o mais amplamente usado. As ligas AZ3I ( $3 \%$ de Al, I\% de Zn e 0,3\% de Mn) são vastamente usadas devido ao fato de oferecerem uma boa combinação de maleabilidade, ductilidade e resistência à corrosão [2].

Em um estudo Cao et al. [3] investigaram o comportamento de corrosão da liga de Mg AZ9I em solução de $\mathrm{NaCl} 0$, I M usando técnicas eletroquímicas e observaram uma natureza altamente corrosiva da liga AZ9| em comparação com o metal Mg. Chang et al. [4] estudaram a influência de elementos de liga como $\mathrm{Zr}$ e Al na resistência à corrosão do $\mathrm{Mg}$ em solução de $\mathrm{NaCl}$ I M usando medições eletroquímicas. Eles observaram que a liga $A Z 9 \mid$ apresentou a pior resistência à corrosão quando comparada às ligas AZ3I, AM60 e ZK60.

As ligas de magnésio para o uso como implantes biodegradáveis vêm sendo bastante pesquisadas [5-7]. As caracterizações envolvem imersão em solução que simula o meio fisiológico ou a simulação em fluidos do corpo tipicamente a $37^{\circ} \mathrm{C}$ favorecendo a produção de filmes óxidos protetivos [8].

A liga AZ9ID foi imersa em solução com 3,5\% de $\mathrm{NaCl}$ a temperatura ambiente por tempos na faixa de I minuto à 72 horas. A corrosão iniciou-se nos limites de grãos dos microconstituintes do eutético e, então, propagou-se através dos grãos alfa primários. Foi observado que a fase beta menos severamente atacadas [9].

Nos tratamentos térmicos das ligas de magnésio, geralmente aplicados com o objetivo de melhorar as propriedades mecânicas da liga, a recristalização pode vir acompanhada pelos processos de autodifusão e interdifusão dos elementos de liga e das fases intermetálicas, dependendo da temperatura, concentração dos elementos de liga $e$ da presença de fases. A liga de magnésio $A Z 3 \mid$ com fase majoritária $\alpha$ - Mg gera a formação de precipitados $\mathrm{Mg}_{17} \mathrm{Al}_{12}$ junto a outros de baixa solubilidade $\left(\mathrm{Al}_{8} \mathrm{Mn}_{5}, \mathrm{Al}_{11} \mathrm{Mn}_{4} \mathrm{e} \mathrm{SiMg}\right)$ na faixa de temperaturas entre 150 e $250^{\circ} \mathrm{C}$. A formação de precipitados pode acarretar considerável endurecimento, mas torna a liga novamente suscetível à corrosão devido a estas fases terem alto potencial de oxidação [7-10].

Tsao [I I ] realizou estudos de resistência à corrosão da liga $A Z 3 \mid$ depois de realizado o seguinte tratamento térmico: I) aquecimento a $420^{\circ} \mathrm{C}$ por $12 \mathrm{~h}$ sob vácuo para dissolver a fase beta $\mathrm{Mg}_{17} \mathrm{Al}_{12} ; 2$ ) resfriamento brusco da liga até a temperatura ambiente; 3 ) envelhecida a $210^{\circ} \mathrm{C}$ por $5 \mathrm{~h}$ e $30 \mathrm{~min}$. Devido provavelmente as dimensões dos precipitados da fase beta, não foi possível visualizar por microscopia óptica a formação desta fase. Neste trabalho foi mostrado que o desaparecimento das maclas depois do envelhecimento a $210^{\circ} \mathrm{C}$ por $5 \mathrm{~h}$ e 30 min melhorou significativamente a resistência à corrosão diminuindo a tendência de formação de pites e a densidade de corrente de corrosão em solução de $\mathrm{NaCl} 3,5 \%(\mathrm{~m} / \mathrm{m})$. Levando se em conta que o tempo de envelhecimento na temperatura de $210^{\circ} \mathrm{C}$ pode proporcionar aumento da resistência à corrosão, foi proposto neste trabalho avaliar o efeito do tratamento térmico a partir da solubilização da amostra em $440^{\circ} \mathrm{C}$ por $24 \mathrm{~h}$ e o envelhecimento em $220^{\circ} \mathrm{C}$ por 4,6 e 12 horas. Portanto, este estudo visa através da polarização cíclica obter dados que permitam fazer uma caracterização do comportamento da corrosão da liga de magnésio AZ3 I assim como uma correlação entre as diferenças microestruturais geradas pelos tratamentos térmicos de solubilização e envelhecimento realizados.

\section{METODOLOGIA}

O objeto de estudo é a liga de magnésio $A Z 3 \mathrm{I}$, que contém $3 \%$ de alumínio e $1 \%$ de zinco, fornecida pela importadora Zincomag na forma de chapa laminada com superfície previamente polida a 800 Mesh. A composição química da liga assim como alguns valores de propriedades mecânicas foi fornecida pelo fabricante e estão apresentadas na Tabela I e Tabela 2, respectivamente.

Quatro amostras da chapa recebida foram cortadas

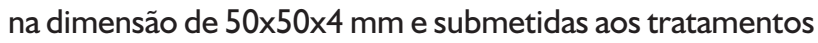
térmicos de solubilização e envelhecimento. As amostras foram solubilizadas a $440^{\circ} \mathrm{C}$ por 24 horas e posteriormente três dessas amostras foram envelhecidas a $220{ }^{\circ} \mathrm{C}$ por 4,6 e 12 horas. O resfriamento após solubilização e após o envelhecimento foi feito em água. Após os tratamentos térmicos as amostras foram lixadas e polidas; as lixas são de $\mathrm{SiC}$ de grana 320 a 2500 mesh, e para polimento sílica coloidal de $0,04 \mu \mathrm{m}$.

Para análise microestrutural após polimento as amostras foram atacadas pelo método de imersão em solução reagente ( $90 \mathrm{ml}$ álcool etílico, $10 \mathrm{ml}$ água destilada, $2 \mathrm{ml}$ ácido acético e 5,8 g ácido pícrico) durante 20 segundos com a finalidade de avaliar a microestrutura predominante, tamanho de grão e presença de fases secundárias possivelmente presentes. As análises via microscopia óptica foram realizadas com o auxílio dos microscópios ópticos Olympus e Nikon LVI50. As medidas de tamanho de grão foram realizadas com auxílio de um perfilômetro baseando na norma ASTM EI I 2 .

Tabela I. Composição química da chapa da liga AZ3I

\begin{tabular}{ccccccccccc}
\hline Elemento & Al & Zn & Mn & Fe & Cu & Ni & Si & Ca & Mg & $\begin{array}{c}\text { Outras } \\
\text { Impurezas }\end{array}$ \\
\hline Peso, \% & $2,5-3,5$ & $0,6-1,4$ & $0,2-1,0$ & 0,003 (máx) & 0,01 (máx) & 0,00 I (máx) & 0,08 (máx) & 0,04 (mpax) & Balanço & 0,30 (máx) \\
\hline
\end{tabular}


A microdureza foi medida com o auxílio do microdurômetro automático Shimadzu HMV-2. Para condução dos testes foi utilizada uma carga de $980 \mathrm{mN}$ aplicada durante 13 segundos. Dez identações foram realizadas em cada amostra da seção transversal da chapa e um intervalo de $95 \%$ de confiança foi obtido por meio da equação I.

$$
\text { Intervalo }(95 \%)=H V(\text { médio }) \pm \frac{1,96(\delta)}{\sqrt{n}}
$$

Onde $\sigma$ é o desvio padrão e $\mathrm{n}$ é o número de medidas realizadas. Para realização dos ensaios eletroquímicos foi utilizada uma célula eletroquímica do tipo Tait com eletrodo de trabalho sendo a liga de magnésio $A Z 3$ I com área de $\mathrm{I} \mathrm{cm}^{2}$, contra eletrodo auxiliar de platina de área de $2 \mathrm{~cm}^{2}$ e eletrodo de referência de prata/cloreto de prata. As medidas foram feitas pelo potenciostato modelo EmSat acoplado ao software OS Trace 4.2. A variação do potencial em circuito aberto ao longo do tempo das amostras em análise foi monitorada por 30 minutos. A curva de Tafel foi determinada a partir da polarização cíclica após a medida do

Tabela 2. Propriedades Mecânicas da chapa da Liga AZ3 I

\begin{tabular}{lc}
\hline Limite de Resistência (Mpa) & 270 \\
Limite de Escoamento (Mpa) & 180 \\
Alongamento (\%) & 15 \\
\hline
\end{tabular}

potencial em circuito aberto, sob ajuste de taxa potencial de varrição de $\mathrm{I} \mathrm{mV} / \mathrm{s}$, iniciando a - $150 \mathrm{mV}$ do potencial em circuito aberto até $+150 \mathrm{mV}$ acima deste mesmo potencial. Todas as medidas foram realizadas em solução aerada em estado estático. A solução utilizada foi $3,5 \% \mathrm{NaCl}$ de $\mathrm{pH} 7$, preparada com água deionizada a temperatura ambiente.

A taxa de corrosão TC foi calculada a partir das equações 2 e 3 de acordo com a norma ASTM GI 02 [12].

$$
E W=\Sigma 1 /(n i f i / w i)
$$

Onde ni é a valencia do $i_{\text {th }}$ elemento na liga, $f i$ a fração mássica do $i_{\text {th }}$ elemento na liga, e wi o peso atômico do $i_{\text {th }}$ elemento na liga.

$$
T C=\left(k x i_{c o r r} x E W\right) / \rho
$$

Onde $k$ é 0,00327 para obter valores em $\mathrm{mm} / \mathrm{ano}, i_{\text {corr }}$ a densidade de corrosão, e $\rho$ a densidade da liga.

\section{RESULTADOS}

A Figura I apresenta a evolução da microestrutura da liga com relação aos tratamentos térmicos realizados. O tamanho de grão medido para cada amostra assim como os valores de microdureza estão apresentados na Tabela 3. a)

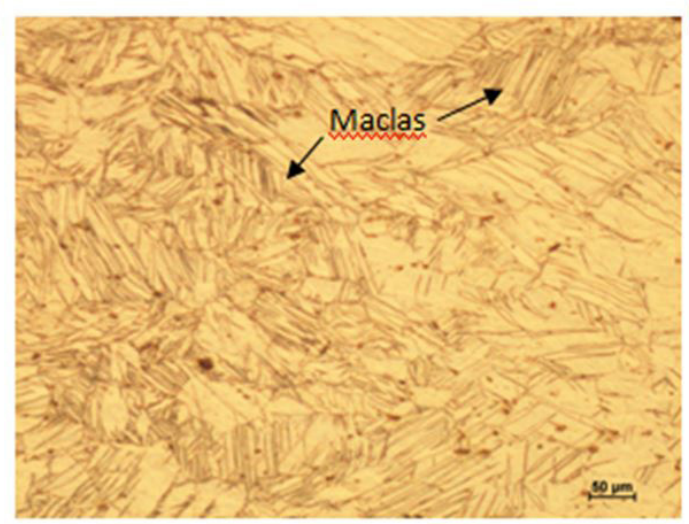

c)

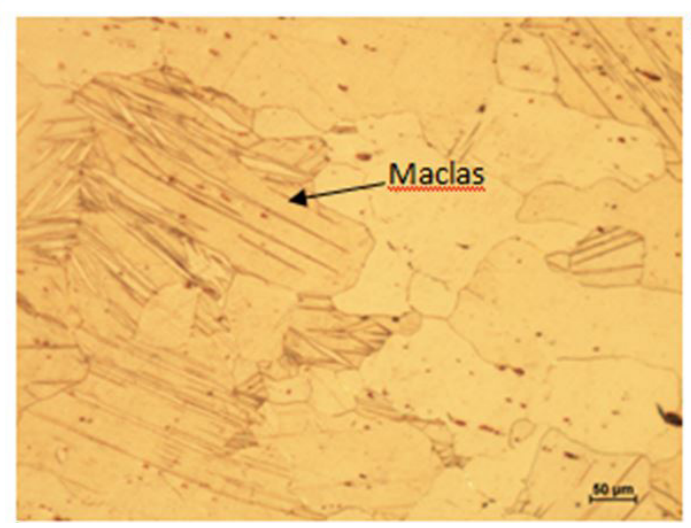

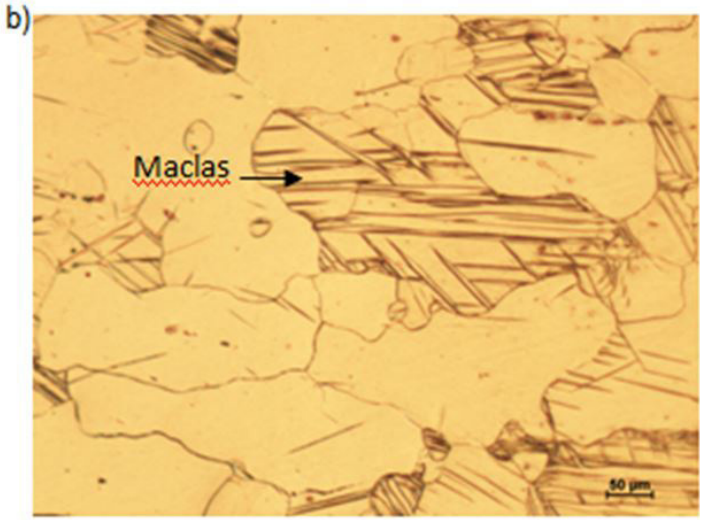

d)

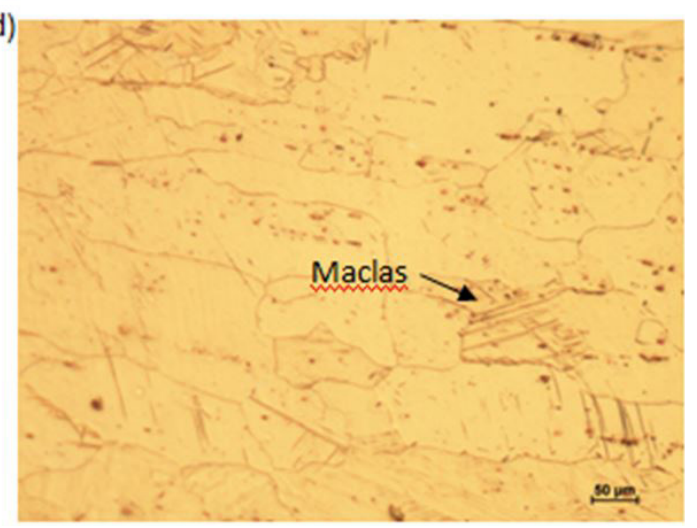

Figura I. Micrografias da seção longitudinal da liga de magnésio AZ3 I (Aumento de 100x); a) Amostra I solubilizada por $24 \mathrm{~h}$ a $440{ }^{\circ} \mathrm{C}$; b) Amostra 2 solubilizada e envelhecida por $4 \mathrm{~h}$ a $220^{\circ} \mathrm{C}$; c) Amostra 3 solubulizada e envelhecida por $6 \mathrm{~h}$ a $220^{\circ} \mathrm{C}$; d) Amostra 4 solubilizada e envelhecida por $12 \mathrm{~h}$ a $220^{\circ} \mathrm{C}$.

Tecnol. Metal. Mater. Miner., São Paulo, 
Na figura 2 é mostrado o potencial em circuito aberto (Open Circuit Potencial - Ocp) para a amostra 2. Pode ser observado um aumento do potencial em circuito aberto nos primeiros segundos e depois o decaimento exponencial do potencial até ficar constante com o tempo de imersão em solução indicando que o processo corrosivo atingiu o estado estacionário. Foram encontrados resultados similares de potencial em circuito aberto vs. tempo para as outras amostras. A partir deste potencial em circuito aberto foram realizados os ensaios de curvas de polarização cíclicas.

Tabela 3. Evolução da Microdureza e Tamanho de Grão da Liga Tratada Termicamente

\begin{tabular}{lcc}
\hline & Microdureza (HV) & Tamanho de Grão $(\mu \mathrm{m})$ \\
\hline Amostra 2 & $31,42+/-4,22$ & $12,09+/-2,92$ \\
Amostra 3 & $33,40+/-7,11$ & $12,25+/-2,46$ \\
Amostra 4 & $37,55+/-5,73$ & $26,39+/-7,47$ \\
\hline
\end{tabular}

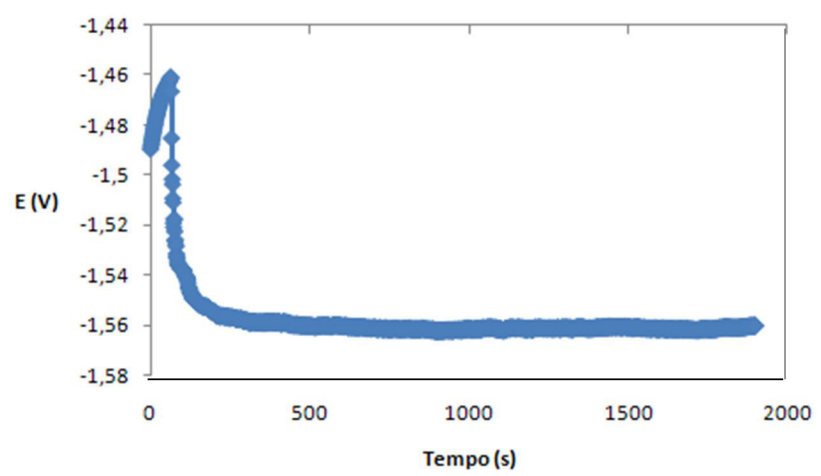

Figura 2. Medida do Potencial em Circuito aberto versus Tempo da amostra 2 imersa em solução de $\mathrm{NaCl} 3,5 \%$.

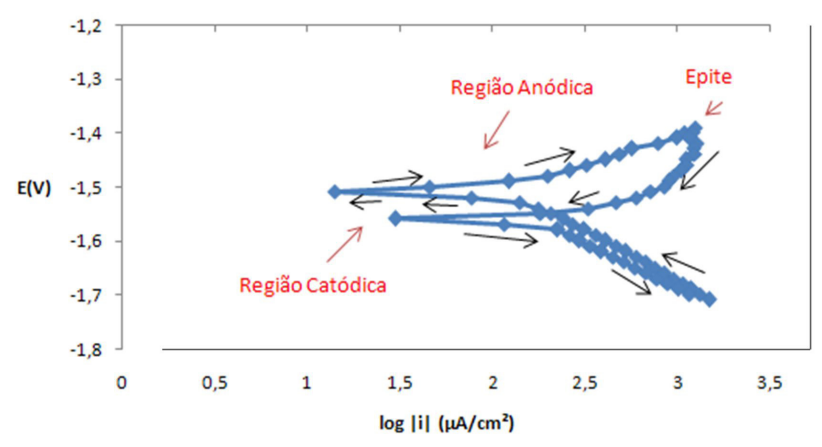

Figura 3. Curva de Polarização Cíclica da amostra 2 imersa em solução de $\mathrm{NaCl} 3,5 \%$.
$\mathrm{Na}$ figura 3 é mostra a curva de polarização cíclica para a amostra 2. Os comportamentos das curvas de polarização das outras amostras foram também similares ao da amostra 2.

A partir dos dados obtidos pelo gráfico do Ocp e das curvas de polarização obteve-se pela extrapolação de Tafel os parâmetros de corrosão apresentados na Tabela 4 .

\section{DISCUSSÃO}

Na Figura la é observada, após o tratamento de solubilização a $440{ }^{\circ} \mathrm{C}$ por 24 horas, uma solução sólida supersaturada com grande presença de maclas que podem ter sido induzidas pelo próprio tratamento térmico [13]. Como a composição da liga $A Z 3$ I é semelhante à $A Z 9$ I, é relatado que o processo de precipitação do $A Z 3$ I envelhecido é semelhante à liga $A Z 9 \mid$ e o precipitado predominante é a fase $\beta-\mathrm{Mg}_{17} \mathrm{Al}_{12}[10,14]$. De acordo com o diagrama de fase deste sistema [15] provavelmente os constituintes são formados por meio dos tratamentos realizados, entretanto estes precipitados são muito finos e em proporções pequenas impossibilitando identificá-los por meio de microscopia óptica. Wan et al. [10], usando tratamentos térmicos similares conseguiram visualizar estas estruturas apenas através de SEM devido as dimensões de tais fases.

As Figuras I b- Id apresentam as microestruturas da liga de magnésio $A Z 3$ I envelhecida, que foram mantidas a $220^{\circ} \mathrm{C}$ por 4,6 e I $2 \mathrm{~h}$, respectivamente. Nestas micrografias é observada a presença de maclas, porém mais dispersas na matriz, e o aumento do tamanho de grão na amostra envelhecida a $220^{\circ}$ em função do tempo de envelhecimento.

Devido as dimensões da fase $\beta-\mathrm{Mg}_{17} \mathrm{Al}_{12}$, ela não pude ser detecta. Mesmo assim o aumento da microdureza da liga (tabela 3) em função do tempo de envelhecimento pode estar correlacionado com uma maior presença desta fase. Ainda de acordo com Wan et al. [10] durante o processo de envelhecimento, $\circ \beta-\mathrm{Mg}_{17} \mathrm{Al}_{12}$ inicialmente precipita no contorno de grão. Com o aumento do tempo de envelhecimento, a fração volumétrica da precipitação continua a aumentar e a precipitação ocorre do interior dos grãos até os contornos. Além disso, de acordo com a literatura [16] a fase $\beta$ melhora a resistência mecânica e a dureza da liga.

De acordo com os estudos anteriores $[6,7,9,17]$, a presença de fase $\beta$ aumenta a diferença de potencial que é responsável pela geração de células galvânicas locais, favorecendo a corrosão. Diferentes reações podem estar

Tabela 4. Parâmetros de Corrosão para Amostras da Liga AZ3 I

\begin{tabular}{|c|c|c|c|c|c|}
\hline & Ocp (mV) & Ecorr $(\mathrm{mV})$ & Epite (mV) & TC (mm/ano) & icorr $\left(\mu \mathrm{A} / \mathrm{cm}^{2}\right)$ \\
\hline Amostra I & $-1555,00$ & $-1482,00$ & $-1400,00$ & 3,18 & $\mathrm{I}, 38 \mathrm{E}+02$ \\
\hline Amostra 2 & $-1556,00$ & $-1505,00$ & $-1390,00$ & 2,56 & $\mathrm{I}, \mathrm{IIE}+02$ \\
\hline Amostra 3 & $-|56|, 00$ & $-1511,00$ & $-1400,00$ & $\mathrm{I}, 86$ & $8,06 \mathrm{E}+0 \mathrm{I}$ \\
\hline Amostra 4 & $-1560,00$ & $-1470,00$ & $-1360,00$ & $|, 0|$ & $4,4|E+0|$ \\
\hline
\end{tabular}


envolvidas na corrosão das ligas de magnésio. A reação geral pode ser expressa pela equação 4 [6]:

$$
\mathrm{Mg}+2 \mathrm{H}_{2} \mathrm{O} \rightarrow \mathrm{Mg}(\mathrm{OH})_{2}+\mathrm{H}_{2}
$$

Contudo, esta reação não explica todos os estágios envolvidos no fenômeno da corrosão deste metal. A suscetibilidade à corrosão das ligas de magnésio está associada à natureza do filme de óxido naturalmente formado presente nas suas superfícies e também relacionado aos elementos de liga catódicos presentes em sua matriz. $\mathrm{Na}$ liga $\mathrm{Mg}-\mathrm{Al}-\mathrm{Zn}$ o $\mathrm{Al}$ e Zn são os principais elementos catódicos que determinam a corrosão das ligas.

Com relação ao potencial em circuito aberto apresentado na Figura 2, todas as amostras apresentaram perfil praticamente idênticos, demonstrando no início do monitoramento um deslocamento para valores mais negativos de potencial tendendo à estabilização após 30 minutos de exposição à solução de $3,5 \%$ de $\mathrm{NaCl}$.

A curva de polarização cíclica mostrada na Figura 3 representa a relação entre o potencial de eletrodo aplicado e a corrente correspondente medida no potenciostato. Esta curva de polarização não é mais representativa de apenas uma única reação, mas sim do efeito global de todas as reações que ocorrem simultaneamente sobre o eletrodo [18]. Devido ao perfil das curvas terem sido semelhantes, apenas uma delas foi utilizada para representar as demais. Contudo, a Tabela 4 apresenta os valores para cada amostra obtidos através dessas curvas.

Observa-se pela Figura 3 que as amostras apresentaram tendência a corrosão por pite, pois após a inversão do potencial a corrente de corrosão diminuiu. As regiões anódicas e catódicas, assim como o ponto onde se identifica o Epite estão indicados na curva.

Avaliando a Tabela 4, percebe-se que não houve considerável variação entre os valores de Ecorr para cada amostra. Logo, os tratamentos térmicos realizados não influenciaram na resistência a corrosão da liga. Na mesma tabela, pode ser visto que a densidade de corrente de corrosão decresce com o aumento do tempo de envelhecimento da liga. Isto tem correlação com os valores apresentados de taxa de corrosão, que também diminui com o aumento do tempo do tratamento térmico.

\section{CONCLUSÃO}

- Na análise da microestrutura observou-se a presença de maclas em todas as amostras estudadas, havendo presença mais acentuada na amostra solubilizada por 24 horas a $440{ }^{\circ} \mathrm{C}$. Não foi possível evidenciar a presença da fase $\beta-\mathrm{Mg}_{17} \mathrm{Al}_{12}$ por microscopia ótica.

- Houve aumento do tamanho de grão na amostra envelhecida a $220^{\circ}$ por 12 horas com relação às amostras envelhecidas por 4 e 6 horas.

- A medida de microdureza apresentou valores similares nas amostras envelhecidas a $220^{\circ} \mathrm{C}$ por 4 e 6 horas. Houve um pequeno aumento na microdureza da amostra envelhecida por 12 horas na mesma temperatura. A presença da fase $\beta-\mathrm{Mg}_{17} \mathrm{Al}_{12}$ pode causar aumento da dureza da liga de magnésio.

- Todas as amostras apresentaram tendência a corroer por meio de pite.

- Houve correlação entre os valores Ocp e Ecorr medidos para cada amostra, não ocorrendo variação significativa entre sí, o que indica que os tratamentos térmicos realizados não interferiram na resistência a corrosão da liga.

- Observou que a taxa de corrosão diminuiu com o aumento do tempo de envelhecimento. Este decréscimo deve-se a diminuição da densidade de corrosão com o aumento dos tempos de envelhecimento das amostras estudadas.

\section{Agradecimentos}

Agradeço imensamente a todos que participaram dos desafios advindos da realização deste trabalho e por tudo que tenho conquistado até então.

Ao Programa de Pós-Graduação em Engenharia Metalúrgica da UFF (PPGEM-UFF).

À CAPES pela concessão de bolsa de estudos (2017-20।8).

\section{REFERÊNCIA}

I Thirumalaikumarasamy D, Shanmugam K, Balasubramanian V. Comparison of the corrosion behaviour of AZ3 IB magnesium alloy under immersion test and potentiodynamic polarization test in $\mathrm{NaCl}$ solution. Journal of Magnesium and Alloys. 2014 [acesso em I 2 dec. 2018];2:I-49. Disponível em: www.sciencedirect.com/science/ article/pii/S22 I39567|4000I39.

2 Revie RW. Uhlig's corrosion handbook. 2nd ed. New York: John Wiley \& Sons, Inc.; 2000.

3 Cao $\mathrm{FH}$, Len VH, Zhang Z, Zhang VH, Zhang X. Corrosion behavior of magnesium and its alloy in $\mathrm{NaCl}$ solution. Russian Journal of Electrochemistry. 2007 [acesso em 12 dec. 20 I8];43:837-843. Disponível em: https://link.springer. com/article/I0.II34/SI023 193507070142. 
4 Chang YL, Qin TW, Wang HM, Zhang Z, editors. Comparison of corrosion behaviors of AZ3I, AZ9I, AM60 and ZK60 magnesium alloys. Transactions of Nonferrous Metals Society of China. 2009 [acesso em I 2 dec. 20 I8]: 19:5 17-524. Disponível em: www.sciencedirect.com/science/article/abs/pii/SI 003632608603052.

5 Agarwal S, Curtin J, Duffy B, Jaiswa LS. Biodegradable magnesium alloys for orthopaedic applications: A review on corrosion, biocompatibility and surface modifications. Materials Science and Engineering C. 2016 [acesso em I3 dec. 2018];68:948-963. Disponível em: https://www.sciencedirect.com/science/article/pii/S00796425I 7300506.

6 Esmaily M, Svensson JE, Fajardo S, Birbilis N, Frankel GS, Virtanen S, et al. Fundamentals and advances in magnesium alloy corrosion. Progress in Materials Science. 2017 [acesso em I3 dec. 2018];89:92-193. Disponível em: https://www.sciencedirect.com/science/article/pii/S00796425 I7300506.

7 Tan L, Dong J, Chen J, Yang K. Development of magnesium alloys for biomedical applications: structure, process to property relationship. Materials Technology. 2018 [acesso em I3 dec. 20 I8];33:235-243. http://dx.doi.org/I0. I080/I 0667857.2017.1405890.

8 Ghoneim AA, Fekry AM, Ameer MA. Electrochemical behavior of magnesium alloys as biodegradable materials in Hank's solution. Electrochimica Acta. 2010 [acesso em I3 dec. 2018];55:6028-6035. Disponível em: https://www. sciencedirect.com/science/article/pii/S00 I 34686/000753X

9 Pinela VM, Oliveira LA, Oliveira MCL, Antunes RA. Study of the Corrosion Process of AZ9ID Magnesium Alloy during the First Hours of Immersion in $3.5 \mathrm{wt} . \% \mathrm{NaCl}$ Solution. International Journal of Corrosion. 2018 [acesso em 13 dec. 2018];2018. Disponível em: https://www.hindawi.com/journals/ijc/2018/8785I54/

10 Wan D, Wang J, Wang G, Lin L, Feng Z, Yang G. Precipitation and responding damping behavior of heat-treated AZ3 I magnesium alloy. Chin Shu Hsueh Pao. 2009 [acesso em 12 dec. 20 I8];22: I-6. Disponível em: https://www. sciencedirect.com/science/article/pii/SI006719108600630.

II Tsao LC. Stress-corrosion cracking susceptibility of AZ3 I alloy after varied heat-treatment in $3.5 \mathrm{wt} . \% \mathrm{NaCl}$ solution. International Journal of Materials Research. 2010 [acesso em I2 dec. 20 I8]; I01:9. Disponível em: https:// www.hanser-elibrary.com/doi/abs/I0.3139//46.I I 0394.

12 American Society for Testing and Materials. ASTM GI 02 - 89: Standard practice for calculation of corrosion rate and related information from eletrochemical measurements. ASTM International: West Conshohocken

I3 Singh IB, Singh M, Das S. A comparative corrosion behavior of $\mathrm{Mg}$, AZ3 I and AZ9I alloys in 3.5\%. J. Magnesium Alloy. 2015 [acesso em 12 dec. 2018];3:I4I-I47. Disponível em: https://www.sciencedirect.com/science/article/pii/ S221395671500016X.

14 Stráskáa J, Janečeka M, Čížekb J, Stráskýa J, Hadzima B. Microstructure stability of ultra-fine grained magnesium alloy AZ3 I processed by extrusion and equal-channel angular pressing (EX-ECAP). Materials Characterization. 2014 [acesso em 12 dec. 2018];94:69-79. Disponível em: https://www.sciencedirect.com/science/article/pii/ SI 0445803 I 4001570.

I5 ASM Handbook committee. Alloy Phase Diagrams, ASM International, 2016.

16 Wang C, Deng K, Nie K, Shang S, Liang W. Competition behavior of the strengthening effects in as-extruded AZ9 I matrix: Influence of pre-existed $\mathrm{Mg}_{17} \mathrm{Al}_{12}$ phase. Materials Science and Engineering A. 2016 [acesso em $12 \mathrm{dec}$. 20 18];A656: I02-I I 0. Disponível em: https://www.sciencedirect.com/science/article/abs/pii/S092 I 5093 I6300235.

17 Song GL, Atrens A, Dargusch M. Influence of microstructure on the corrosion of diecast AZ9ID. Corrosion Science. 1999 [acesso em 12 dec. 20 I8];4I:249-273. Disponível em: https://www.sciencedirect.com/science/article/ $\mathrm{pii} / \mathrm{S} 0010938 \times 98001218$.

18 Kleiner S, Uggowitzer PJ. Mechanical Anisotropy of Extruded Mg - 6\% Al - I\% Zn Alloy. Materials Science and Engineering A. 2004 [acesso em I 2 dec. 20 I8];379:258-263. Disponível em: https://www.sciencedirect.com/ science/article/abs/pii/S092I 509304001893

Recebido em: 15 Dez. 2018

Aceito em: 27 Fev. 2019 